\author{
Professor Constantin ANGHELACHE,PhD \\ E-mail: actincon@yahoo.com \\ The Bucharest University of Economic Studies \\ Associate Professor Mădălina-Gabriela ANGHEL,PhD \\ E-mail: madalinagabriela_anghel@yahoo.com \\ "ARTIFEX" University of Bucharest \\ Lecturer Ștefan Virgil IACOB,PhD \\ E-mail: stefaniacob79@yahoo.com \\ "ARTIFEX" University of Bucharest
}

\title{
STATISTICAL-ECONOMETRIC METHODS AND MODELS USED IN THE ANALYSIS OF THE CAPITAL MARKET UNDER THE RISK OF INFLATION
}

\begin{abstract}
In this article, the authors focused on presenting a model for analyzing the accumulation of assets and portfolio decisions taken under the risk of inflation, starting from the fact that the capital market is also influenced by the effect of price changes.

It is analyzed the possibility of expressing the model of accumulation of assets and portfolio decisions taken under the risk of inflation, taking into account the notions regarding the long-term structure and the real yields, aspects regarding the time structure of the nominal yields, as well as the dynamic expression model of this date. of inflation.

It should be borne in mind that the dynamic estimation of inflation helps to establish the dynamics of the stock returns in the optimal final use of some factors that we consider to have an effect on the portfolio market.

Also, we can find that some conclusions are analyzed and drawn regarding the considerations in the allocation of assets in correlation with the inflation horizon. In this regard, the perspective of the dynamic evolution of inflation must be taken into account in order to determine this major influence factor under the risk empire on the prices on the capital market.

Keywords: asset, portfolio, bond market, inflation risk, multifactorial model, stochastic modeling, Ito equation, Wiener stochastic processes.
\end{abstract}

JEL Classification: C13, E22, E31

\section{Introduction}

In this article, the authors started from the study of the model of analysis of the accumulation of assets and the portfolio decisions that should be considered taking into account the risk presented by the evolution of inflation.

The authors are concerned with establishing the intertemporal accumulation model of the assets, considering that they are under the influence of

DOI: 10.24818/18423264/54.2.20.03 
the inflation risk. In this context, the model of intertemporal asset allocation is analyzed in detail, as well as the set of investment opportunitiesthat must be considered. Also, the action of the agents on the capital market is analyzed in the context in which the effect that it can influence has to be taken into account, determines the market under dynamic programming which is closely correlated with the dynamic evolution of the inflation rate.

The way in which the intertemporal portfolio can be built and used in terms of asset evolution and portfolio decisions taken under the influence of inflation is presented. In this context, there are presented aspects regarding notions regarding the long-term structure of real yields, but they were closely correlated with the dynamic effect of the price increase, in other words the inflation rate.

The broad presentation of this situation analyzes the time structure of the nominal yields using concrete data that introduced, used in the regression models or other econometric models give the possibility of an interpretation of the way the capital market evolves.

In other ideas, the model regarding the structure of the dynamics of inflation becomes important, in the sense that it is a determining factor in establishing the assets and portfolios on the capital market. In this context, we can see that it is necessary to estimate the dynamics of inflation so that it is correlated with the estimation of the dynamics of stock returns and finally the optimal use of the factors considered to be able to establish those models that help to avoid the risk of inflation or at least to determine and impose some measures to ensure tempering and controlling the risk of inflation.

Finally, we analyze and present a series of aspects regarding the determination of the parameters of the assets under the conditions of a given risk, that is to say a risk for which we have established a parameter that presents its influence. Also, some considerations are expressed regarding the allocation of assets in correlation with the investment horizon on the capital market, taking into account the inflation evolution perspective.

\section{Literature review}

Amini et al. (2010) analyzed a number of issues regarding the predictability of stock returns. Anghelache and Anghel (2018) studied and presented the main methods and models used in capital market analyzes. Anghelache, Voineagu and Gheorghe (2013) highlighted the fundamental elements regarding the measurement and analysis of inflation. Anghelache, Mitrut and Voineagu (2013) addressed the essential aspects of macroeconomic statistics. Campbell and Thompson (2008) focused on methods of estimating the capital premium. Cooper (2009) studied elements of risk premiums. Ferreira and SantaClara (2011), as well as Hjalmarsson (2010) analyzed methods of estimating the return on the capital market. A similar theme is studied by Lettau and van Nieuwerburgh (2008). Giacomini and Rossi (2010) conducted a comparative study of forecasts in unstable environments. Hsiao and Semmler (2009) studied longterm financial data series. Jarrow and Yildirim (2003) studied the evolution of the

DOI: $10.24818 / 18423264 / 54.2 .20 .03$ 
Statistical-Econometric Methods and Models Used in the Analysis of the Capital Market under the Risk of Inflation

prices of securities under inflation conditions. Markowitz (2010) maintains his point of view on portfolio theory, and Kolm et al (2014) conducted an analysis of the evolution of portfolio theory. Markowitz (2014) addressed issues related to the average variance. Piketty (2014) expressed his view on capital in the 21 st century. Wachter (2003) analyzed the correlation between risk aversion and long-term debt allocation. Welch and Goyal (2008) empirically studied the performance of capital premium forecasting. Wolff (2013) studied the relationship between asset prices and middle-class well-being. Van Binsbergen and Koijen (2010) addressed elements of predictive regression. Zhou (2010) studied the effectiveness of asset pricing models.

\section{Research methodology, data, results and discussions}

\section{Analysis model of the accumulation of assets and portfolio decisions taken under the risk of inflation}

The main investment assets are debt securities, including conventional nominal bonds, as well as inflation-indexed bonds.

The unifactorial model is suitable for the structural analysis of real bonds. The aspect regarding the modeling of the interest rate, must contain two factors, namely the real interest rate and the inflation rate, in order to determine the dynamics of the prices of the nominal bonds.

The Jarrow and Yildirim model extended to a multifactorial one, will contain the real interest rate and the estimated inflation rate as key factors. This model includes the price variables of nominal bonds indexed to inflation. Also, this model distinguishes the forecast inflation from the inflation realized as two different risk factors. The forecasted inflation expresses the perspective on the future inflation whose risk rate is determined on the market of nominal bonds. Inflation captures the uncertainty of changing consumer prices whose risk rate is determined on the market for inflation-indexed bonds.

The model of multifactorial structure is estimated on the basis of market data, of nominal and indexed bonds with inflation, as well as on the stock market. The results of the parameter estimates are subsequently used to build the optimal investment strategy within the intertemporal framework.

We will build a multifactorial model considering the prices of nominal bonds, but also those of inflation. The bond model incorporates a stochastic price index and the two systematic factors (the forecast inflation rate and the real interest rate) that determine the change in the bond markets. The model will include a theoretical basis for solving the problem of building an investment strategy in the conditions of price uncertainty and systematic change factors.

The extension of the continuous asset allocation framework is done taking into account a time-varying $I_{t}$ price index, modeled by the deflation process, according to the relationship:

DOI: 10.24818/18423264/54.2.20.03 
$\frac{d I_{t}}{I_{t}}=\pi_{t} d t+\sigma_{I} d W_{t}^{I}$

where $I_{0}=1, W_{t}^{I}$ is a Wiener process, $\pi_{t}$ is the forecast inflation rate and $\sigma_{I}$ represents the standard deviation. The Wiener process is based on stochastic characteristics, respectively Wiener $[W(0)=0]$; increment process $W(t)-W(s)$, which is independent for $s<t$; the increment process $W(t)-W(s)$ is normally distributed, with zero mean and variancet-s; $W(t)$ is a continuous process, ie $t$ belongs to the set of real numbers R.A price index expresses the price for a fixed basket of goods. The time-varying price index provides two valuation terms: the nominal value and the actual deflated value of the goods.

\section{- Multifactorial model for nominal and inflation-linked bonds}

The study offers a multifactorial bond model. The factors that determine the evolution of the bond markets are the real interest rate and the forecast inflation rate. The bond model may also include a stochastic price index.

\section{- The factors considered in the multifactorial model}

We consider that the real interest rate and the predicted rate of $r_{t}$ inflation are the two factors that determine the price of the nominal bond. The two factors follow the processes of the Gaussian mean form and satisfy the relations:

$d r_{t}=k_{r}\left(\bar{r}-r_{r}\right) d t+g_{r} d W_{t}^{r}$

and

$d \pi_{t}=k_{\pi}\left(\bar{\pi}-\pi_{r}\right) d t+g_{\pi} d W_{t}^{\pi}$

where $W_{t}^{r}$ and $W_{t}^{\pi}$ are correlated with the instantaneous variation $d W_{t}^{r} d W_{t}^{\pi}=$ $\rho_{r \pi} d t$. Both the real interest rate and the forecast inflation rate can record negative values. Therefore we adopt the Gaussian processes instead of quadratic processes for modeling these two factors. All bonds are assumed to be in the exponential affine family - the Duffie-Kan family. equation.

The relations (1), (2) and (3) are stochastic equations of the nature of the Ito

\section{a) The factor of the nominal obligation}

Let $P_{n}\left(r_{t}, \pi_{t}, t, T\right)$ (sometimes we use a short notation $P_{n}(t, T)$ ) if the abbreviation does not cause confusion) denote the nominal obligation of the zero coupon at $t$ with the maturity date $T$. The payment of the nominal bond at maturity is normalized as a monetary unit. The nominal connection relationship has the following form:

$P_{n}\left(r_{t}, \pi_{t}, t, T\right)=\exp \left[-A_{n}(T-t)-B_{n r}(T-t) r_{t}-B_{n \pi}(T-t) \pi_{t}\right]$ and

$P_{n}(t, T)=1$

The coefficients $A_{n}(\tau), B_{n}(\tau) \operatorname{and} B_{n \pi}(\tau)$ will be determined by the arbitration conditions. Due to the terminal state (5), the limit values for coefficients are given by the equations: 
Statistical-Econometric Methods and Models Used in the Analysis of the Capital Market under the Risk of Inflation

$A_{n}(0)=0, B_{n}(0)=0, B_{n \pi}(0)=0$

The nominal yield is defined to be:

$Y_{n}(t, T)=\frac{-\ln P_{n}(t, T)}{T-t}=\frac{A_{n}(T-t)}{T-t}+\frac{B_{n r}(T-t)}{T-t} r_{t}+\frac{B_{n \pi}(T-t)}{T-t} \pi_{t}$

The instantaneous nominal interest rate $R_{t}$ is defined as the instantaneous yield expressed by the relation:

$R_{t}=\lim _{T \rightarrow t} Y_{n}(t, T)$

If we apply this last result to the yield formula (7), it results in an equation of the instantaneous nominal interest rate $R_{t}$, which has the following form: $R_{t}=A_{n}^{\prime}(0)+B_{n r}^{\prime}(0) r_{t}+B_{n \pi}^{\prime}(0) \pi_{t}$ where $A^{\prime}$ denotes the derivative of $A$.

\section{b) The bond factor indexed with inflation}

Let $P_{I}(t, T)$ indicate the price IIB (zero coupon - Inflation Indexed Bonds) at $t$ and maturity at $T$.The payment at maturity is made in units of money $I_{T}$, so that:

$P_{I}(T, T)=I_{T}$

There is a real bond $P_{t}(t, T)=\frac{P_{I}(t, T)}{I_{t}}$ that is corresponding $P_{I}(t, T)$ with its valued price in the cargo unit. At maturity, the real bond $P_{r}(T, T)=1$ represents a unit of goods. We assume that this is only affected by the instantaneous real interest rate, and its price follows the dynamics given by the relationship: $P_{r}\left(r_{t}, t, T\right)=\exp \left[-A_{r}(T-t)-B_{r r}(T-t) r_{t}\right]$

The condition of the terminal (10) implies the condition of delimiting the coefficients:

$$
A_{r}(0)=0, B_{r r}(0)=0
$$

The real yield is the interest rate of the real bond, defined as:

$Y_{t}(t, T)=\frac{-\ln P_{r}(t, T)}{T-t}=\frac{A_{r}(T-t)}{T-t}+\frac{B_{r r}(T-t)}{T-t} r_{t}$

There is a real money account on the market, which gives the real face value, respectively:

$M_{I}(t)=M_{r}(t) I_{t}$

where the money consumption account is an account with real accumulations (records):

$$
M_{r}(t)=\exp \left(\int_{0}^{t} r_{s} d s\right)
$$

\section{Intertemporal accumulation of assets at risk of inflation - model used \\ - The building the model of intertemporal allocation of assets}

If we use the programmed dynamic approach, we can solve the problem of intertemporal decision. Suppose that there are identical agents, which are defined by $V_{0}$ units of capital at the nominal value at time zero and they try to maximize the estimated final utility at time $T$, according to the relation:

DOI: 10.24818/18423264/54.2.20.03 
$\max _{\alpha_{t}, t \in[0, T]} e_{0}\left[U\left(v_{t}\right)\right]$

If we consider the uncertainty about the price, we will introduce the term $v_{t}$, representing the real capital, adjusted to the price level $v_{t}=\frac{V_{t}}{I_{t}}$. The established utility is a constant function of relative risk aversion (CRRA), according to the relation:

$U\left(c_{t}\right)=\frac{c_{t}^{1-\gamma}}{1-\gamma}$

where $\gamma>0$ represents the risk aversion coefficient

- The systematic factors of the model regarding intertemporal accumulation of assets at risk of inflation

We specify that the systematic factors, which determine the change of the yield dynamics of the bonds influenced by the instantaneous real interest rate $r_{t}$ and the forecast inflation rate $\pi_{t}$. We use $X_{t}$ to name these factors so that the equality of $X_{t}=\left(r_{t}, \pi_{t}\right)^{T}$ is satisfied. The dynamic factors expressed in vector form lead to the relation:

$d X_{t}=F_{t} d t+G_{t} d W_{t}^{X}$

where the functions $F$ and $G$ are defined by the relations:

$F=\left(\begin{array}{c}k_{r}\left(\bar{r}-r_{r}\right) \\ k_{\pi}\left(\bar{\pi}-\pi_{r}\right)\end{array}\right), \quad G=\left(\begin{array}{cc}g_{r} & 0 \\ 0 & g_{\pi}\end{array}\right)$

We also have $W_{t}^{X}=\left(W_{t}^{r}, W_{t}^{\pi}\right)^{T}$ and the correlation matrix, which is synthesized in the equation:

$R_{X X} d t=d W_{t}^{X} d W_{t}^{X T}$

\section{- The presentation of investment opportunities}

We consider that the set of investment opportunities includes five assets: two nominal bonds with different maturities $T_{1}, T_{2}$, one IIB with maturity at $\mathrm{T}_{3}$, the financial nominal account and a stock. Suppose the stock price follows a geometric progression, respectively:

$\frac{d P_{S}(t)}{P_{S}(t)}=\left(R_{t}+\lambda_{S} \sigma_{S}\right) d t+\sigma_{S} d W_{t}^{S}$

with $\sigma_{s}>0$, a constant instantaneous standard evolution of stock returns and $\lambda_{S}>$ 0 a constant price of the market risk associated with the uncertainty $W_{t}^{S}$.

Synthesizing all the returns of the risk of the asset according to the relation (21), we obtain the following vector form:

$\left.\begin{array}{l}\left(\begin{array}{c}d P_{n}\left(t, T_{1}\right) / P_{n}\left(t, T_{1}\right) \\ d P_{n}\left(t, T_{2}\right) / P_{n}\left(t, T_{2}\right) \\ d P_{I}\left(t, T_{3}\right) / P_{n}\left(t, T_{3}\right)\end{array}\right)=\mu_{t} d t+\sum_{t} d W_{t}, \\ d P_{S}(t) / P_{n}(t)\end{array}\right)=$ 
Statistical-Econometric Methods and Models Used in the Analysis of the Capital Market under the Risk of Inflation

$$
\begin{aligned}
& \Sigma_{t}=\left(\begin{array}{ccc}
-B_{n r}\left(T_{1}-t\right) g_{r}-B_{n \pi}\left(T_{1}-t\right) g_{\pi} & 0 & 0 \\
-B_{n r}\left(T_{1}-t\right) g_{r}-B_{n \pi}\left(T_{1}-t\right) g_{\pi} & 0 & 0 \\
-B_{r r}\left(T_{1}-t\right) g_{r} & 0 & 0 \\
0 & 0 & \sigma_{S}
\end{array}\right) \\
& \underline{1}=\left(\begin{array}{l}
1 \\
1 \\
1 \\
1
\end{array}\right), \quad d W_{t}=\left(\begin{array}{c}
d W_{t}^{r} \\
d W_{t}^{\pi} \\
d W_{t}^{I} \\
d W_{t}^{S}
\end{array}\right)
\end{aligned}
$$

Which lead to the final relationship:

$$
\Lambda_{t}=\Lambda_{0}+\Lambda_{1}\left(\begin{array}{l}
r_{t} \\
\pi_{t}
\end{array}\right) \operatorname{cu} \Lambda_{0}=\left(\begin{array}{l}
\lambda_{0} \\
l_{0} \\
\lambda_{I} \\
\lambda_{S}
\end{array}\right), \Lambda_{1}=\left(\begin{array}{cc}
\lambda_{1} & 0 \\
0 & l_{1} \\
0 & 0 \\
0 & 0
\end{array}\right)
$$

The relationship (22) is maintained as a result of the arbitration conditions and the dynamics of the stock return (21). The four risk factors $d W_{t}^{r}, d W_{t}^{\pi}, d W_{t}^{I}, d W_{t}^{S}$ are correlated with the correlation matrix $R_{A A} d t=$ $d W_{t} d W_{t}^{T}$. We will note this correlation matrix between $W_{t}$ and $W_{t}^{I}$ with $R_{A I} d t=$ $d W_{t} d W_{t}^{I}$.

\section{- The effect of the agents' action in the market}

The agents can review their investment decision $\alpha_{t}$ without trading costs for any time $t \in[0, T]$, where $\alpha_{t}=\left(\alpha_{i t}\right)_{i=1, \ldots, 4}$, each $\alpha_{i t}$ represents the proportion of investment in the risky asset $i$. The sum of the investment must be balanced by the nominal financial account $M_{n}(t)$, so that its ratio $\alpha_{0 t}$ is equal to $\alpha_{0 t}=1-$ $\sum_{i=1}^{4} \alpha_{i t}$. Considering the decisions $\alpha_{t}$ the capital evolves dynamically, according to the relation:

$\frac{d V_{t}}{V_{t}}=\sum_{i=0}^{4} \alpha_{i t} \frac{d P_{i t}}{P_{i t}}=R_{t} d t+\alpha_{t}^{T}\left[\left(\mu_{t}-R_{t} \underline{1}\right) d t+\sum_{t} d W_{t}\right]$

where $\alpha_{t}^{T}=\left(\alpha_{1 t}, \ldots, \alpha_{4 t}\right), \mu_{t}$ is the expected yield vector $\sum_{t}$ and is the volatility matrix. In order to obtain the evolution of the real capital $v_{t}=\frac{V_{t}}{I_{t}}$ for the beginning we apply the Lema inverse relation of the price index process and obtain:

$d\left(\frac{1}{I_{t}}\right)=\frac{1}{I_{t}}\left(-\pi_{t} d t+\sigma_{I}^{2} d t-\sigma_{I} d W_{t}^{I}\right)$

We apply the Lema relation again to $v_{t}=\frac{V_{t}}{I_{t}}$ and using the result of the evolution of the nominal capital (26) we will obtain the evolution of the dynamics of the real capital, according to the relation:

$\frac{d v_{t}}{v_{t}}=\left(R_{t}-\pi_{t}+\sigma_{I}^{2}\right) d t+\alpha_{t}^{T}\left(\mu_{t}-R_{t} \underline{1}-\sigma_{I} \sum_{t} R_{I}\right) d t+\alpha_{t}^{T} \sum_{t} d W_{t}-\sigma_{I} d W_{t}^{I}(27)$

Finally, the problem of the investment decision of the agents is to determine the optimal path $\alpha_{t}$ for $t \in[0, T]$, which maximizes the objective function under the real budget constraint (27) and the dynamics of the factors.

DOI: 10.24818/18423264/54.2.20.03 
- Significant considerations regarding the dynamic programming approach

Considering $J\left(t, T, v_{t}, X_{t}\right)$ by which we formalize the function of the value over a subperiod $[t, T]$, with the initial real capital given by $v_{t}$ and the state of the factor $X_{t}$, that is:

$J\left(t, T, v_{t}, X_{t}\right)=\max _{\alpha_{S}, s \in[t, T]} e_{t}\left[U\left(v_{T}\right)\right]$

The key result of the dynamic programming approach is the value function that must satisfy the Hamilton-Jacobi-Bellman equation, according to the relation:

$J\left(t, T, v_{t}, X_{t}\right)=\max _{\alpha_{t}}\left\{J\left(t+d t, T, v_{t+d t}, X_{t+d t}\right)\right\}$

$0=\max _{\alpha_{t}}\left\{\left[R_{t}-\pi_{t}+\sigma_{I}^{2}+\alpha_{t}^{T}\left(\mu_{t}-R_{t} \underline{1}-\sigma_{I} \sum_{t} R_{A I} \sigma_{I}\right)\right] J_{v} v_{t}+\frac{1}{2}\left(\alpha_{t}^{T} \sum_{t} R_{A A} \sum_{t}^{T} \alpha_{t}-\right.\right.$ $\left.2 \sigma_{I} \alpha_{t}^{T} \sum_{t} R_{A I}+\sigma_{I}^{2}\right) J_{v v} v_{t}^{2}+\left(\alpha_{t}^{T} \sum_{t} R_{A X} G_{t}^{T}-\sigma_{I} R_{I X} G_{t}^{T}\right) J_{v X} v_{t}+F_{t}^{T} J_{X}+$ $\left.\frac{1}{2} \sum_{i, j=1}^{2} G_{i t} R_{X X} G_{j t}^{T} J_{X_{i} X_{j}}+\frac{\partial}{\partial t} J\right\}$

where $\quad R_{X A} d t=d W_{t}^{X} d W_{t}^{T}, R_{A I} d t=d W_{t} d W_{t}^{T}, R_{X I} d t=d W_{t}^{X} d W_{t}^{I} \quad$ and $\quad G_{i t}$ denotes row $i$ in the $G_{t}$ matrix and represents the partially relevant derivative.

It follows that the optimal portfolio $\alpha_{s}, s \in[t, T]$ is independent of the initial capital level $v_{t}$ because from the CRRA utility function, we deduce that the dynamics $\frac{d v_{s}}{v_{s}}$, is independent of $d X_{s}$. This aspect is summarized in the relation:

$J\left(t, T, v_{t}, X_{t}\right)=v_{t}^{1-\gamma} e^{-\delta T} \max _{\alpha_{s}, t \leq s \leq T} e_{t}\left[U\left(\frac{v_{T}}{v_{t}}\right)\right]=v_{t}^{1-\gamma} J\left(t, T, v_{t}, X_{t}\right)$,

and thus we can decompose $J\left(t, T, v_{t}, X_{t}\right)$ into:

$J\left(t, T, v_{t}, X_{t}\right)=e^{-\delta t} U\left(v_{T}\right) \phi\left(t, T, X_{t}\right)^{\gamma}$,

where $\phi\left(t, T, X_{t}\right)^{\gamma}=e^{-\delta t}(1-\gamma) J\left(t, T, 1, X_{t}\right)$

The boundary condition is given by equality:

$\phi\left(T, T, X_{t}\right)=1$,

because $J\left(T, T, V_{t}, X_{t}\right)=e^{-\delta t} U\left(v_{T}\right)$ is in accordance with its definition (28).

Applying the condition for the first command $\alpha_{t}$ we obtain the expression of the optimal $\alpha_{t}$ in terms $J$ and $\phi$ :

$\alpha_{t}^{*}=\left(\sum_{t} R_{A A} \sum_{t}^{T}\right)^{-1}\left(-\frac{J_{v} v_{t}}{J_{v v} v_{t}^{2}}\left(\mu_{t}-R_{t} \underline{1}\right)-\frac{1}{J_{v v} v_{t}^{2}} \sum_{t} R_{A X} G_{t}^{T} J_{v X} v_{t}+\right.$ $\left.\frac{J_{v} v_{t}+J_{v v} v_{t}^{2}}{J_{v v} v_{t}^{2}} \sigma_{I} \sum_{t} R_{A I}\right)=\left(\sum_{t} R_{A A} \sum_{t}^{T}\right)^{-1}\left(\frac{1}{\gamma}\left(\mu_{t}-R_{t} \underline{1}\right)+\sum_{t} R_{A X} G_{t}^{T} \frac{\phi_{X}}{\phi}+(1-\right.$ $\left.\left.\frac{1}{\gamma}\right) \sigma_{I} \sum_{t} R_{A I}\right)=\left(\sum_{t}^{T}\right)^{-1}\left(\frac{1}{\gamma} R_{A A}^{-1} \sum_{t}^{-1}\left(\mu_{t}-R_{t} \underline{1}\right)+R_{A A}^{-1} \sum_{t} R_{A X} G_{t}^{T} \frac{\phi_{X}}{\phi}-\frac{1-\gamma}{\gamma} R_{A A}^{-1} R_{A I} \sigma_{I}\right)$

The optimal intertemporal portfolio can be broken down into: the efficient portfolio of medium variability, the term of intertemporal coverage and the term of covering the inflation. 
Statistical-Econometric Methods and Models Used in the Analysis of the Capital Market under the Risk of Inflation

The optimal allocation of the portfolio is determined by the three advantages presented above in the relation (34), adjusted with the possible effect of the risks $\sum_{t} R_{A A} \sum_{t}^{T}$.

The first benefit is the excess yield $\mu_{t}-R_{t} \underline{1}$. Since this portfolio takes into account the distribution between the forecast yield and the risk only at the present moment $\left(\sum_{t} R_{A A} \Sigma_{t}^{T}\right)^{-1}$, it is also called static portfolio.

The second advantage is given by the term of intertemporal hedging, with which an investor can increase the future forecast utility, using the correlation between a systematic beneficial factor and the asset shocks. For example, the interest rate, if the current level of the factor increases, will increase the optimal final utility $J\left(t, T, v_{t}, X_{t}\right)$ in the future. The term of intertemporal coverage highlights that the investor increases the ownership of an asset if it is positively correlated with the profit factor.

In mathematical terms there is the relation $\phi_{X}>0$ for the profit factor. The investor should hold more of an asset if the correlation between the asset and the $R_{A X}$ factor is positive.

The third advantage corresponds to a term for hedging inflation. It can be decided to further increase the expected future utility by protecting against price uncertainty. Price uncertainty is represented by a variation $\sigma_{I}$ equal to zero of the price shocks. I found that in relation (34), if there is no uncertainty regarding the price $\sigma_{I}=0$, then there is no need for inflation coverage.

Applying the solution (34) of the HJB equation (34), we find that the HJB equation is transformed, having the form, from the relation:

$$
\begin{aligned}
& 0=\frac{\partial}{\partial t} \phi+F_{t}^{T} \phi_{X}+\left(\frac{1-\gamma}{\gamma} G_{t} R_{X A} R_{A A}^{-1} \sum_{t}^{-1}\left(\mu_{t}-R_{t} \underline{1}\right)-\frac{(1-\gamma)^{2}}{\gamma} G_{t} R_{X A} R_{A A}^{-1} R_{A I} \sigma_{I}-\right. \\
& \left.(1-\gamma) G_{t} R_{X I} \sigma_{I}\right)^{T} \phi_{X}+\frac{1}{2} \sum_{i, j=1}^{n} \phi_{X_{i} X_{j}} G_{i t} R_{X X} G_{j t}^{T}+ \\
& \frac{1-\gamma}{2 \phi} \sum_{i, j=1}^{n} \phi_{X_{i}} \phi_{X_{j}} G_{i t}\left(R_{X A} R_{A A}^{-1} R_{A X}-R_{X X}\right) G_{j t}^{T}+\phi\left(-\frac{\delta}{\gamma}+\frac{1-\gamma}{\gamma}\left(R_{t}-\pi_{t}+\sigma_{I}^{2}\right)+\right. \\
& \frac{1-\gamma}{2 \gamma^{2}}\left(\mu_{t}-R_{t} \underline{1}\right)^{T}\left(\sum_{t} R_{A A} \sum_{t}^{T}\right)^{-1}\left(\mu_{t}-R_{t} \underline{1}\right)+\frac{(1-\gamma)^{3}}{2 \gamma^{2}} \sigma_{I}^{2} R_{I A} R_{A A}^{-1} R_{A I}-\frac{(1-\gamma)^{2}}{\gamma^{2}}\left(\mu_{t}-\right. \\
& \left.\left.R_{t} \underline{1}\right)^{T} \sum_{t}^{T-1} R_{A A}^{-1} R_{A I} \sigma_{I}-\frac{1-\gamma}{2} \sigma_{I}^{2}\right)
\end{aligned}
$$

\section{- The analysis and resolution of the intertemporal portfolio 1}

We use Liu's method to solve the HJB equation (35). Brennan and Xia and Munk et al. does not apply directly to the analyzed case. Although Liu does not consider price uncertainty, equation (35) still falls in the retraction class of the quadratic assets defined later, because the additional terms in equation (35) are generated by price uncertainty.

The solution $\phi\left(t, T, X_{t}\right)$ satisfies the equation $X_{t}=\left(r_{t}, \pi_{t}\right)^{T}$ and is given by:

$$
\phi\left(t, T, X_{t}\right)=\exp \left(c(\tau)+b(\tau)^{T} X_{t}+\frac{1}{2} X_{t}^{T} Q(\tau) X_{t}\right), c u \tau=T-t
$$

\section{DOI: $10.24818 / 18423264 / 54.2 .20 .03$}


The function $Q(\tau)$ is a matrix function $2 \times 2$, there is the vector function $b(\tau)$, and $2 \times 1$ is a scalar function $c(\tau)$. The functions satisfy the equations:

$\frac{d}{d \tau} Q=M_{1}^{T} Q+Q M_{1}+Q h_{0} Q+\frac{1-\gamma}{2 \gamma^{2}} H_{2}$

$\frac{d}{d \tau} b=M_{1}^{T} b+Q h_{0} b+Q M_{2}+\frac{1-\gamma}{2 \gamma^{2}} H_{1}+\frac{1-\gamma}{\gamma} \delta_{1}$

$\frac{d}{d \tau} c=M_{2}^{T} b+\frac{1}{2} b h_{0} b+\frac{1}{2} \operatorname{Tr}\left(h_{0} Q\right)+\frac{1-\gamma}{2 \gamma^{2}} H_{0}+\frac{1-\gamma}{\gamma} \delta_{0}-\frac{\delta}{\gamma}$

with

$M_{1}=\left(\begin{array}{cc}-k_{r}+\frac{1-\gamma}{\gamma} g_{r} \lambda_{1} & 0 \\ 0 & -k_{\pi}+\frac{1-\gamma}{\gamma} g_{\pi} \lambda_{1}\end{array}\right)$

$M_{2}=\left(\begin{array}{l}-k_{r} \bar{r}+\frac{1-\gamma}{\gamma} g_{r}\left(\lambda_{0}-\sigma_{I} \rho_{I r}\right) \\ -k_{\pi} \bar{\pi}+\frac{1-\gamma}{\gamma} g_{r}\left(\lambda_{0}-\sigma_{I} \rho_{I \pi}\right)\end{array}\right)$

$H_{1}=2 \Lambda_{0}^{T} R_{A A}^{-1} \Lambda_{1}$

$H_{0}=\Lambda_{0}^{T} R_{A A}^{-1} \Lambda_{1}+\sigma_{I}^{2}-2(1-\gamma) \lambda_{I} \sigma_{I}$

$\delta_{1}=\left(\begin{array}{l}1 \\ 0\end{array}\right)$ și $\delta_{0}=-\sigma_{I} \rho_{I}$

The boundary conditions are given by the relations:

$Q(0)=0, b(0)=0$ și $c(0)=0$

is given by the relation:

We will specify that the elasticity, with respect to the factor $X_{t}=\left(r_{t}, \pi_{t}\right)^{T}$,

$\frac{\phi_{X}(t, T, X)}{\phi(t, T, X)}=b(T-t)+Q(T-t) X$

with $\phi_{X}(t, T, X)=\frac{d}{d X} \phi(t, T, X)$. The functions $b$ and $Q$ satisfy relations (38), (39) and boundary conditions.

Applying the analytical solution of the factor elasticity to the portfolio formula, we can determine the optimal portfolio in an analytical form.

The set of investment opportunities is given by the relation (21) and consists of two nominal bonds, one IIB and one stock. Let $\left(\begin{array}{l}\Psi_{1}(\tau, X) \\ \Psi_{2}(\tau, X)\end{array}\right)=\frac{\phi_{X}(t, T, X)}{\phi(t, T, X)}$ indicate the elasticity of the factor (46). The optimal allocation of the portfolio with the three components decomposition is given by the equality:

$\alpha_{t}^{*}=\alpha_{t}^{(M)}+\alpha_{t}^{(I)}+\alpha_{t}^{(P)}$

where:

$\alpha_{t}^{(M)}=\frac{1}{2}\left(\sum_{t}^{T}\right)^{-1} R_{A A}^{-1} \Lambda_{t}$ 
Statistical-Econometric Methods and Models Used in the Analysis of the Capital Market under the Risk of Inflation

$\alpha_{t}^{(I)}=\left(\begin{array}{c}D^{-1}\left(-B_{n \pi}\left(\tau_{2}\right) \psi_{2}(\tau, X)+B_{n r}\left(\tau_{2}\right) \Psi_{2}(\tau, X)\right) \\ D^{-1}\left(B_{n \pi}\left(\tau_{1}\right) \psi_{1}(\tau, X)-B_{n r}\left(\tau_{1}\right) \Psi_{1}(\tau, X)\right) \\ 0 \\ 0\end{array}\right)$

and

$\alpha_{t}^{(P)}=\left(1-\frac{1}{\gamma}\right)\left(\begin{array}{c}-D^{-1}\left(B_{n \pi}\left(\tau_{2}\right) B_{r r}\left(\tau_{3}\right)\right) \\ D^{-1}\left(B_{n \pi}\left(\tau_{1}\right) B_{r r}\left(\tau_{3}\right)\right) \\ 1 \\ 0\end{array}\right)$

with $\tau=T-t ; \quad \tau_{i}=T_{i}-t$, for $i=1,2,3$ și $D=\left(\begin{array}{l}B_{n r}\left(\tau_{2}\right) B_{n r}\left(\tau_{2}\right) \\ B_{n \pi}\left(\tau_{1}\right) B_{r \pi}\left(\tau_{2}\right)\end{array}\right)$

The considered solutions can determine the optimal portfolio in analytical form and the set of investment opportunities.

3. Estimating the model of asset accumulation and portfolio decisions taken under the risk of inflation

- The main concepts regarding the long-term structure of real yields

The first step of the estimation procedure is that of the instantaneous real rate through the real returns of the market. Conventional data analyzed consist of real daily yields with maturation horizons at 5, 7 and 10 years (the data for the period 2009-2018 are processed by the authors). The time series of the real yields are shown in Figure 1:

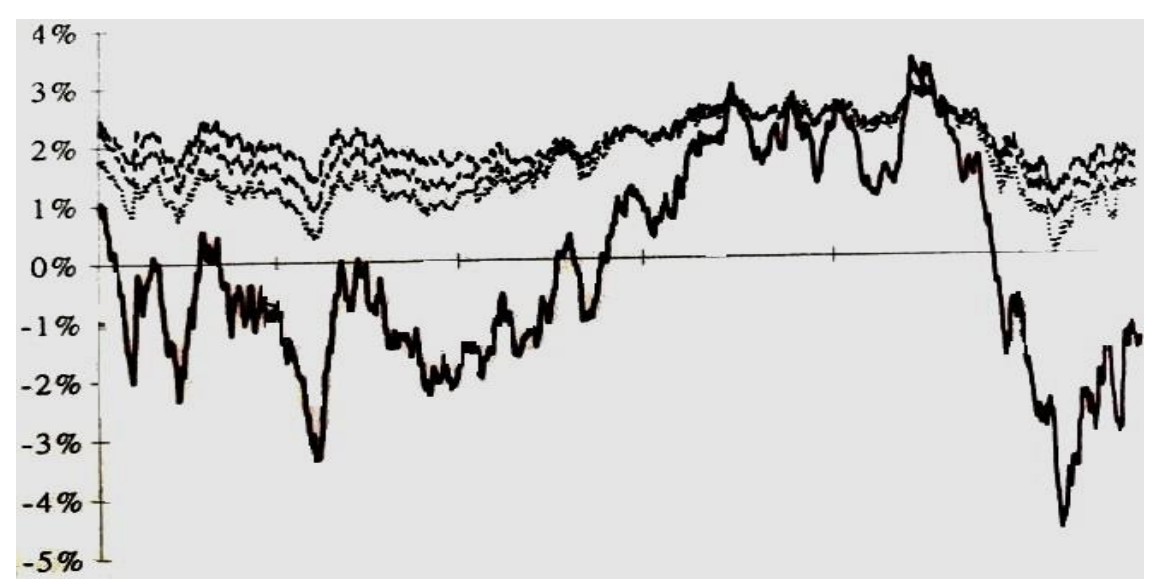

Figure 1. Real TIPS yields and estimated real rate during the period Jan 2009Jan 2018

DOI: $10.24818 / 18423264 / 54.2 .20 .03$ 
By implementing the Kalman filter, the equation to be observed is the real efficiency formula, where the coefficients $A_{r}(\tau)$ and $B_{r r}(\tau)$ have been calculated with measurement errors. The observation equation becomes:

$Y_{r}\left(t, t+\tau, r_{t}\right)=\frac{A_{r}(\tau)}{\tau}+\frac{B_{r r}(\tau)}{\tau}+\epsilon_{t}^{\tau}$

where $\epsilon_{t}^{\tau}$ expresses the measurement error which is independent and identical normally distributed, with the zero mean and the variation of the real rate $\epsilon_{t}^{\tau}$, which is given by the relation:

$r_{t+\Delta}=e^{-k_{r} \Delta} r_{t}+\bar{r}\left(1-e^{-k_{r} \Delta}\right)+\sigma_{r} u_{r t}$

where the innovation term $u_{r t}$ follows a standard normal distribution and the volatility $\sigma_{r}=\sqrt{\frac{g_{r}^{2}}{2 k_{r}}\left(1-e^{-2 k_{r} \Delta}\right)}$. Because the exact solution is available, the Kalman filter is made without any error. The results of the estimation of the parameters are shown in table 1 , and the estimated real interest rate $r_{t}$ is presented in figure 1.

Table 1. The estimates of real yields and their statistics

\begin{tabular}{|c|c|c|c|}
\hline \multirow{2}{*}{\multicolumn{2}{|c|}{$\tilde{k}_{r}$ (sensitivity) }} & & $t_{\text {stat. }}$ \\
\hline & & $\begin{array}{l}\text { Estimated } \\
0.5044 \\
\end{array}$ & 25.54 \\
\hline \multicolumn{2}{|l|}{$\bar{r}$} & -0.0019 & -1.18 \\
\hline \multicolumn{2}{|l|}{$g_{r}$} & 0.0208 & 47.10 \\
\hline \multicolumn{2}{|l|}{$\lambda_{0}^{*}=\lambda_{0}-\sigma_{I} \rho_{I r}$} & -0.6768 & -15.17 \\
\hline \multicolumn{2}{|l|}{$\sigma_{\in^{\tau}}$} & 0.0009 & 67.10 \\
\hline \multicolumn{2}{|l|}{$k_{r}$ (return) } & $=\tilde{k}_{r}$ & Tabel 2 \\
\hline$\tau$ & 5 years $\%$ & 7 years $\%$ & 10 years $\%$ \\
\hline Average & 1.54 & 1.79 & 1.99 \\
\hline SD & 0.61 & 0.46 & 0.35 \\
\hline$\frac{A_{r}(\tau)}{\tau}$ (level) & 1.61 & 1.84 & 2.03 \\
\hline$\frac{B_{r r}(\tau)}{\tau}$ (sensitivity) & 36.50 & 27.50 & 19.70 \\
\hline 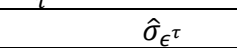 & 0.09 & 0.05 & 0.11 \\
\hline$\frac{\hat{\sigma}_{\epsilon^{\tau}}}{S D}$ & 14.70 & 10.20 & 32.70 \\
\hline
\end{tabular}

The market price of the risk $\Lambda_{t}^{r}$ is extended by including a linear term $\Lambda_{1} r_{t}$. Under these conditions, the parameter $k_{r}$ determines the inversion rate of $r_{t}$, while another separate parameter, $\tilde{k}_{r}=\lambda_{r}-\lambda_{1} g_{r}$, which appears in $B_{r r}(\tau)$, deals with the sensitivity of the yield. For the real rate, $k_{r}$ is accepted to be identical to $\tilde{k}_{r}$ according to the Likelihood Ratio (LR) test as shown in Table 2:

Table 2. LR test for $\boldsymbol{H}_{\mathbf{0}}: \boldsymbol{k}_{\boldsymbol{r}}=\widetilde{\boldsymbol{k}}_{\boldsymbol{r}}$

\begin{tabular}{|l|l|l|l|}
\hline No restrictions $H_{0}$ & $H_{0}: k_{r}=\tilde{k}_{r}$ & P-value - LR test & Result \\
\hline $23,187.88$ & $23,178.54$ & $45.21 \%$ & $H_{0}: k_{r}=\tilde{k}_{r}$ accepted \\
\hline
\end{tabular}

The instantaneous real rate $r_{t}$ is an average recovery process with an inversion parameter $k_{r}=0.5044$, which corresponds to a half time of 1.4 years. It has a negative average $\bar{r}=-0.19 \%$, which is not significantly different from zero. 
Statistical-Econometric Methods and Models Used in the Analysis of the Capital Market under the Risk of Inflation

The trajectory of the estimated real rate is shown in figure 1 . The real rate was present in the negative area at the beginning, then a tendency of constant growth follows.

From the modeling perspective, this growth is supported by a positive evolution of the real empirical yields and the convergence of the term spread in that period.

The model formation is significant. The row $\frac{\widehat{\sigma}_{\epsilon} \tau}{S D}$ in table 1 shows that over $70 \%$ of the total variation can be explained by this model.

\section{- Some aspects regarding the time structure of the nominal yields}

The second step of the estimation procedure is to deduce the forecast inflation rate. The required data are the yields of the nominal bonds and the real rate estimated at the first step. The consumer price index achieved is not necessary in this estimation, which correlates the nominal bond markets with the real ones. The market data of the nominal yields are calculated based on the declarations of nominal bonds in the securities market.

We obtain daily nominal yields with time until the maturity of one month, three and six months, respectively one year, two, three, five, seven, ten and twenty years. The nominal yields are shown in Figure 2:

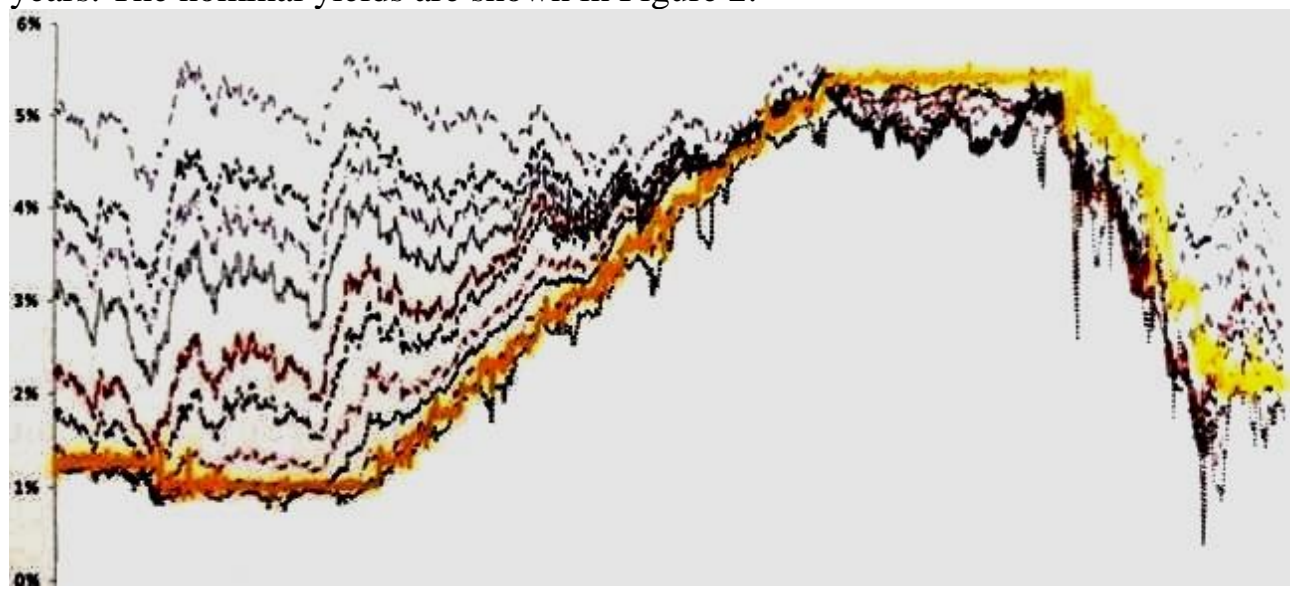

Figure 2. The nominal bond yields and allocated funds rateduring the period Jan 2009-Jan 2018

During this period the economy went through different stages of the economic cycles. There was also an overheating on the mortgage market, due to an economic decline.

The equation is based on the yield formula with the measurement error $\epsilon_{t}^{\tau}$ resulting in:

$Y_{r}\left(t, t+\tau, r_{t}, \pi_{t}\right)=\frac{A_{n}(\tau)}{\tau}+\frac{B_{n r}(\tau)}{\tau}+\frac{B_{n \pi}(\tau)}{\tau}+\epsilon_{t}^{\tau}$, 
where the innovation term $A_{n}(\tau), B_{n r}(\tau)$ and $B_{n \pi}(\tau)$ follows a standard normal distribution and volatility $\epsilon_{t}^{\tau}$.

There is an identification problem in the estimate. We will perform the estimation by setting $\bar{\pi}=0$. Next, we substitute $\pi_{t}$ with $\bar{\pi}$ estimated from the realized inflation rate.

The iterative process begins with fixing an initial value of parameter $\rho_{r \pi}$, after which we estimate the other parameters using the maximum likelihood method. After estimation, we make the correlation of the resulting sample, $\rho_{r \pi}^{(1)}=$ $\left[\hat{u}_{r t}, \hat{u}_{\pi t}\right]$, where $\hat{u}_{r t}$ and $\hat{u}_{\pi t}$ are the residuals. We compare $\hat{\rho}_{r \pi}^{(0)}$ with $\hat{\rho}_{r \pi}^{(1)}$ to determine if they have a deviation of up to $5 \%$, in which case the iteration process stops. Otherwise, we expect $\rho_{r \pi}$ equal to $\hat{\rho}_{r \pi}^{(1)}$ and repeat the process.

Provided the estimation model is true and the maximum likelihood sample is constant, the iteration scheme provides a consistent estimator.

The market price of the risk $\Lambda_{t}^{\pi}$ is extended to a linear term $l_{1} \pi_{t}$. The parameter $k_{\pi}$ determines the inversion speed of $\pi_{t}$, while another separate parameter, $\tilde{k}_{\pi}=\lambda_{\pi}+$ $l_{1} g_{\pi}$, which appears in $B_{r \pi}(\tau)$, determines the sensitivity of the output. For the forecast inflation rate the hypothesis $k_{\pi}=\tilde{k}_{\pi}$ is rejected according to the Probability Ratio (LR) test presented in Table 3 . The estimates are summarized in Table 4.

Table 3. LR test for $\boldsymbol{H}_{\mathbf{0}}: \boldsymbol{k}_{\boldsymbol{r}}=\widetilde{\boldsymbol{k}}_{\boldsymbol{r}}$

\begin{tabular}{|l|l|l|l|}
\hline No restrictions $H_{0}$ & $H_{0}: k_{r}=\tilde{k}_{r}$ & P-value - LR test & Result \\
\hline $62,311.69$ & $62,100.00$ & $4.95 \times 10^{-94}$ & $H_{0}: k_{r}=\tilde{k}_{r}$ respins \\
\hline
\end{tabular}

\begin{tabular}{|c|c|c|c|c|c|}
\hline \multicolumn{6}{|c|}{ Table 4:.Estimates of nominal yields and their statistics } \\
\hline & & \multicolumn{2}{|c|}{ Estimated } & \multicolumn{2}{|l|}{$t_{\text {stat }}$} \\
\hline$\tilde{k}_{\pi}$ (sensitivity) & & \multicolumn{2}{|c|}{0.7523} & \multicolumn{2}{|l|}{34.65} \\
\hline$g_{\pi}$ & & \multicolumn{2}{|c|}{0.0171} & \multicolumn{2}{|l|}{81.35} \\
\hline$l_{0}$ & & \multicolumn{2}{|c|}{0.4456} & \multicolumn{2}{|l|}{2.97} \\
\hline$\xi_{0}^{0}$ & & \multicolumn{2}{|c|}{0.0322} & \multicolumn{2}{|l|}{9.67} \\
\hline$\hat{\sigma}_{\tau}$ & & \multicolumn{2}{|c|}{0.0029} & \multicolumn{2}{|l|}{74.97} \\
\hline \multicolumn{2}{|l|}{$k_{\pi}$ (return) } & \multicolumn{2}{|c|}{1.7475} & \multicolumn{2}{|l|}{17.64} \\
\hline \multirow[t]{2}{*}{$\rho_{r \pi}$} & & \multicolumn{2}{|c|}{-0.6903} & & \\
\hline & 1 month $\%$ & 3 months $\%$ & 6 months $\%$ & 1 year $\%$ & 2 years $\%$ \\
\hline Average & 2.76 & 2.86 & 3.02 & 3.11 & 3.28 \\
\hline SD & 1.57 & 1.58 & 1.59 & 1.47 & 1.25 \\
\hline$\frac{A_{r}(\tau)}{\tau}$ (level) & 3.24 & 3.29 & 3.35 & 3.48 & 3.69 \\
\hline$\frac{B_{r r}(\tau)}{\tau}$ (sensitivity) & 96.93 & 91.16 & 83.35 & 70.28 & 51.70 \\
\hline$\hat{\sigma}_{\epsilon^{\tau}}$ & 0.33 & 0.20 & 0.16 & 0.16 & 0.30 \\
\hline$\frac{\hat{\sigma}_{\epsilon^{\tau}}}{S D}$ & 20.88 & 12.41 & 10.31 & 10.70 & 24.30 \\
\hline$\tau$ & 3 years $\%$ & 5 years $\%$ & 7 years $\%$ & 10 years $\%$ & 20 years $\%$ \\
\hline Average & 3.46 & 3.82 & 4.07 & 4.33 & 4.86 \\
\hline SD & 1.07 & 0.75 & 0.57 & 0.42 & 0.30 \\
\hline$\frac{A_{r}(\tau)}{\tau}$ (level) & 3.86 & 4.11 & 4.27 & 4.41 & 4.59 \\
\hline
\end{tabular}


Statistical-Econometric Methods and Models Used in the Analysis of the Capital Market under the Risk of Inflation

\begin{tabular}{|c|l|l|l|l|c|}
\hline$\frac{B_{r r}(\tau)}{\tau}$ (sensitivity) & 39.67 & 25.97 & 18.89 & 13.29 & 6.65 \\
\hline$\hat{\sigma}_{\epsilon^{\tau}}$ & 0.35 & 0.29 & 0.26 & 0.24 & 0.44 \\
\hline$\frac{\hat{\sigma}_{\epsilon^{\tau}}}{S D}$ & 32.92 & 38.57 & 45.65 & 58.30 & 148.08 \\
\hline
\end{tabular}

The upper area of Table 4 provides the parameter estimates from the nominal yield formulas. The lower area provides information about the standard deviation of the yields, the estimates for the coefficients $\frac{A_{r}(\tau)}{\tau} s_{i} \frac{B_{r r}(\tau)}{\tau}$ of the relation (3) and the estimated mean square error.

The average return parameter $k_{\pi}=1.7475$ indicates that the dynamic $\pi_{t}$ is stationary with a short half-decay of 0.4 years (approximately 5 months). The estimated trajectory $\pi_{t}$ is shown in figure 3 . The average $\bar{\pi}$ de $\pi_{t}$ is taken from the inflation rate obtained from table 5 . The correlation between the estimated inflation rate $\pi_{t}$ and the real rate $r_{t}$, is negative $-69.03 \%$. According to the classic MundellTobin effect, the expected inflation is inversely proportional to the expected real interest rate.

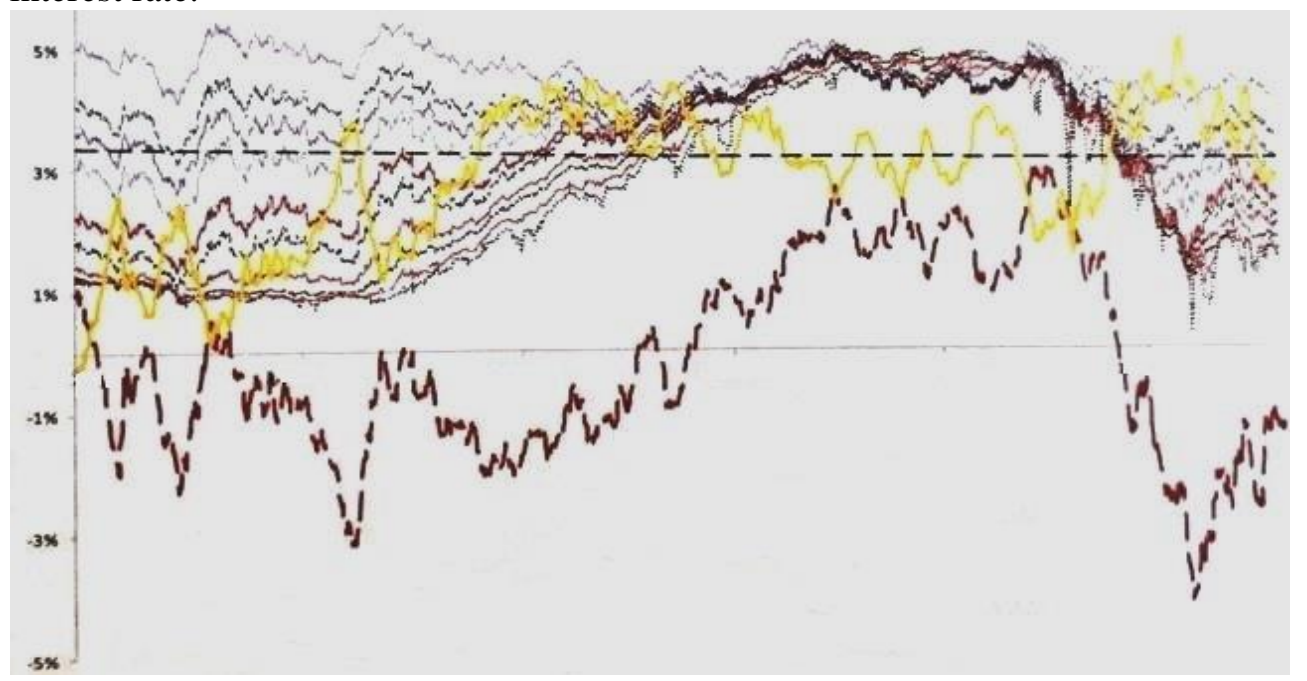

Figure 3. The nominal returns and estimated factorsduring the period Jan 2009-Jan 2018

Table 5. The results of estimates

\begin{tabular}{|c|l|l|}
\hline & Estimated & $t_{\text {stat }}$ \\
\hline$k_{\pi}$ & 1.7479 & 2.03 \\
\hline$g_{\pi}$ & 0.0000 & 0.00 \\
\hline $\bar{\pi}$ & 0.0336 & 5.63 \\
\hline$\sigma_{I}$ & 0.0141 & 12.45 \\
\hline
\end{tabular}

The sensitivity parameter in figure $4 \tilde{k}_{\pi}=0.7523$ indicates a low sensitivity of the yield of the nominal bonds for the forecast inflation, as shown in

DOI: 10.24818/18423264/54.2.20.03 
row $\frac{B_{r r}(\tau)}{\tau}$ of table 4 . Similar to the real yields, the yield differences of nominal bonds began to converge after a period of time. This convergent period is accompanied by a high level of the real interest rate.

The model constitution is reasonable as shown on row $\frac{\widehat{\sigma}_{\epsilon^{\tau}}}{S D}$ of table 4. Errors are below 30\% for bond yields up to three years and around 50\% for those with a maturity up to decade. The yield on 20 -year bonds has a high ratio, as it has very little variation.

As a validation check of the model estimate, we compare the instantaneous nominal interest rate based on the estimation results and the corresponding market interest rates. The comparison is shown in Figure 4, where we found that the solution is satisfactory only up to a certain point.

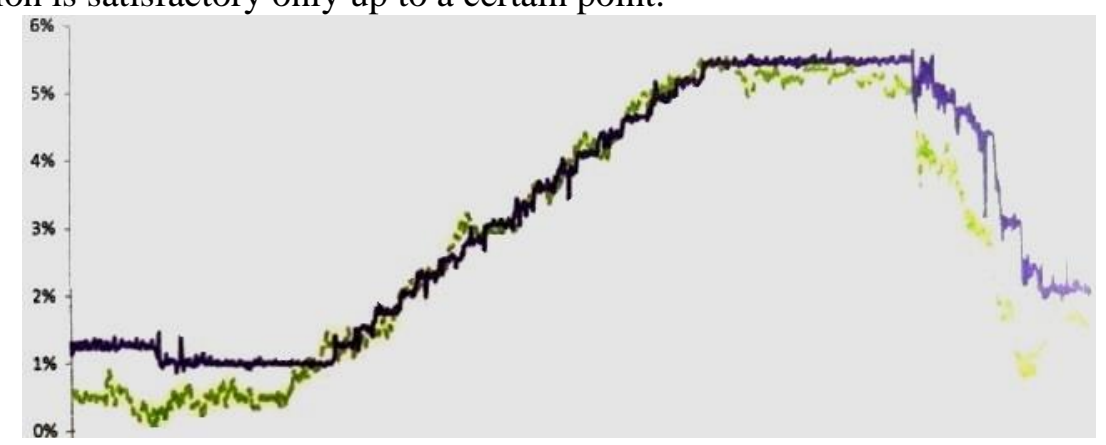

Figure 4. The national fund rate and the instant estimated rateduring the period Jan 2009-Jan 2018

Due to the mentioned extension, we cannot directly apply the solution methods to this case and we adopt the Liu type solution method, which can solve the allocation of assets for a rather dynamic class of them.

The main investment assets are debt securities, including conventional nominal bonds, as well as inflation-indexed bonds. Jarrow and Yildirim propose a clear way to price these two bonds in a single model that satisfies the constraint without arbitrage. They adopt the "exchange rate" view in which the nominal obligations in the "nominal world", those in the "real world" are correlated with the price index, given by a "exchange rate".

\section{Conclusions}

This article emphasizes that the capital market must be analyzed taking into account the inflation rate factor that has a special effect on the analysis of portfolios, the accumulation of assets, the decisions of portfolios taken on the market taking into account the risk of inflation.

Most of the issues that have been analyzed by the authors are modeled using some statistical-econometric methods, based on which to establish the possibility of interpreting and analyzing the multiple aspects that indicate 
Statistical-Econometric Methods and Models Used in the Analysis of the Capital Market under the Risk of Inflation

accumulation of assets and taking portfolio decisions in the context of the inflation rate.

The study performed theoretically expresses the analysis method to be interpreted and these are brought and formulated as statistical-econometric models expressed by mathematical relationships based on which the parameters can be calculated and the evolution of the capital market can be identified under the risk of inflation.

However, macroeconomic data are expressed in real terms, in the sense that they are deflated, that is, they are brought to the actual figures that exist under the influence of the factors we consider. These statistical-econometric relations are also analyzed in the light of the use of data based on which evolution trends are expressed, such as the federal fund rate and the instant estimated rate, the nominal returns and the estimated factors that have been analyzed and so on.

In order to facilitate the understanding of how statistical and econometric methods and models can be used in the analysis of the capital market under the influence of inflation risk, we have presented a series of data in the form of series and also made a graphical representation of these developments in order to identify more easily how this data is required.

A final conclusion is that the risk of inflation is a very important factor, which must be taken into account in the analyses carried out in relation to the dynamic evolving capital market.

\section{REFERENCES}

[1] Amini, S. et al. (2010), Stock Return Predictability Despite Low Autocorrelation. Economics Letters, 108: 101-103;

[2] Anghelache, C., Anghel, M.G. (2018), Econometrie generală. Teorie și studii de caz.Economică Publishing, Bucureşti;

[3] Anghelache, C., Voineagu, V., Gheorghe, M. (2013), Metode și modele de măsurare şi analiză a inflaţiei. Economică Publishing, Bucureşti;

[4] Anghelache, C., Mitruţ, C., Voineagu, V. (2013), Statistică macroeconomică. Sistemul Conturilor Naţionale. Economică Publishing,, Bucureşti;

[5] Campbell, J.V., Thompson, S.B. (2008), Predicting the Equity Premium out of Sample: Can Anything Beat the Historical Average. Review of Financial Studies, 21(4): 1509-1531;

[6] Cooper, I. (2009), Time-Varying Risk Premiums and the Output Gap. Review of Financial Studies, Society for Financial Studies, 22(7): 2601-2633;

[7] Ferreira, M.A., Santa-Clara, P. (2011), Forecasting Stock market Returns: The Sum of the Parts is more than the Whole. Journal of Financial Economics, 100; 514-537;

DOI: $10.24818 / 18423264 / 54.2 .20 .03$ 
ConstantinAnghelache, Mădălina-Gabriela Anghel, Ștefan Virgil Iacob

[8] Giacomini, R., Rossi, B. (2010), Forecast Comparisons in Unstable Environments. Journal of Applied Econometrics, John Wiley \& Sons, Ltd., 25(4): 595-620;

[9] Hjalmarsson, E. (2010), Predicting Global Stock Returns. Journal of Financial and Quantitative Analysis, Cambridge University Press, 45(1): 4980 ;

[10] Hsiao, C.Y., Semmler, W. (2009), Harmonic Analysis of Long Run Financial Data, Working Paper;

[11] Jarrow, R., Yildirim, Y., (2003), Pricing Treasury Inflation Protected Securities and Related Derivations Using an HJM Model.Journalof Financial and Quantitative Analysis, 38(2): 337-358;

[12] Kolm, P.N. et al. (2014), 60 Years of Portfolio Optimization: Practical Challenges and Current trends. European Journal of Operational Research, 234: 356-371;

[13] Lettau, M., van Nieuwerburgh, S. (2008), Reconciling the Return Predictability Evidence. Review of Financial Studies, 21(4): 1607-1652;

[14] Markowitz, H. (2010), Portofolio Theory: As I still See it.The Annual Review of Financial Economics, 2(1): 1-23;

[15] Markowitz, H. (2014), Mean-variance Approximations to Expected Utility. European Journal of Operational Research, 234: 346-355;

[16] Piketty, T. (2014), Capital in the Twenty-first Century,Boston: Harvard University Press;

[17] Rapach, D.E., Zhou, G. (2013), Forecasting Stock Returns, In Handbooks of economic forecasting (Vol. 2, in press), Elsevier;

[18] Van Binsbergen, J.H., Ralph S.J.K. (2010), Predictive Regressions: A

Present-Value Approach. Journal of Finance, American Finance Association, 65(4): 1439-1471, August;

[19] Wachter, J. (2003), Risk Avesion and Allocation to Long-term Bonds. Journal of Economic Theory, 112: 325-333;

[20] Welch, I., Goyal, A. (2008), A Comprehensive Look at the Empirical Performance of Equity Premium Prediction.Review of Financial Studies, 21(4), 1455-1508;

[21] Wolff, E. (2013), The Asset Price Meltdown and the Wealth of the Middle Class.NBER Working Paper No. 18559;

[22] Zhou, G. (2010), How Much Stock Return Predictability Can We Expect from an Asset Pricing Model?. Economics Letter, 108, 184-186.

DOI: $10.24818 / 18423264 / 54.2 .20 .03$ 